\title{
Phase-locking to a free-space terahertz comb for metrological-grade terahertz lasers
}

\author{
L. Consolino ${ }^{1,2}$, A. Taschin ${ }^{1,2}$, P. Bartolini', S. Bartalini, ${ }^{1,2}$, P. Cancio ${ }^{1,2}$, A. Tredicucci ${ }^{3}$, H. E. Beere ${ }^{4}$, \\ D. A. Ritchie ${ }^{4}$, R. Torre ${ }^{1,5}$, M. S. Vitiello 2,3 \& P. De Natale ${ }^{1,2}$
}

Optical frequency comb synthesizers have represented a revolutionary approach to frequency metrology, providing a grid of frequency references for any laser emitting within their spectral coverage. Extending the metrological features of optical frequency comb synthesizers to the terahertz domain would be a major breakthrough, due to the widespread range of accessible strategic applications and the availability of stable, high-power and widely tunable sources such as quantum cascade lasers. Here we demonstrate phase-locking of a $2.5 \mathrm{THz}$ quantum cascade laser to a free-space comb, generated in a $\mathrm{LiNbO}_{3}$ waveguide and covering the 0.1-6 THz frequency range. We show that even a small fraction $(<100 \mathrm{nW})$ of the radiation emitted from the quantum cascade laser is sufficient to generate a beat note suitable for phase-locking to the comb, paving the way to novel metrological-grade terahertz applications, including high-resolution spectroscopy, manipulation of cold molecules, astronomy and telecommunications.

\footnotetext{
${ }^{1}$ LENS, European Laboratory for NonLinear Spectroscopy, Via N. Carrara 1, Sesto Fiorentino (FI) I-50019, Italy. ${ }^{2}$ INO, Istituto Nazionale di Ottica - CNR, Largo E. Fermi 6, Firenze I-50125, Italy. ${ }^{3}$ NEST, Istituto Nanoscienze - CNR and Scuola Normale Superiore, Piazza San Silvestro 12, Pisa I-56127, Italy. ${ }^{4}$ Cavendish Laboratory, University of Cambridge, J J Thomson Avenue, Cambridge CB3 OHE, UK. ${ }^{5}$ Dipartimento di Fisica e Astronomia, Università di Firenze, Via G. Sansone 1, Sesto Fiorentino, Firenze 1-50019, Italy. Correspondence and requests for materials should be addressed to L.C. (email: luigi.consolino@ino.it).
} 
M etrological-grade, high-power, terahertz sources offer great perspectives for applications in many different fields. Besides their straightforward use for high-resolution and high-sensitivity molecular gas spectroscopy ${ }^{1,2}$ and far-infrared astronomy ${ }^{3}$, a number of disciplines could benefit from their actual availability. In biophysics, for example, narrow and stable terahertz radiation can help understand complex dynamics problems, in principle addressing high-precision molecular recognition and protein folding ${ }^{4}$. Similarly, absolute-frequency-controlled, high-power terahertz sources can be a key tool for controlling cold molecular ensembles ${ }^{5}$.

Terahertz-emitting metrological sources, widely and continuously frequency-tunable, date back to the early $1980 \mathrm{~s}^{6,7}$, and have recently evolved to systems based on photomixing techniques ${ }^{8,9}$. They are based on either microwave or infrared standards, ensuring a very high spectral purity and an absolute frequency reference, but a drawback is that their typical powers (above $1 \mathrm{THz}$ ) are at the nanowatt level. This has hindered, until now, the deployment of terahertz sources for experiments and applications requiring higher optical powers.

In this context, extension of optical frequency comb synthesizers (FCSs) to the terahertz domain is a key progress. Optical FCSs have indeed represented a major improvement to frequency metrology ${ }^{10,11}$, separating the roles of light source and reference and providing, in principle, a frequency ruler for any laser emitting within their spectral range. Their huge potential is exploitable by directly beating the laser with one of the FCS teeth, and by detecting the beat note with a square-law detector. The test of maturity for frequency combs has been, indeed, the achievement of power levels suitable for an efficient detection of the beating signal with standard-grade detectors $^{12}$.

Several approaches have been recently pioneered to migrate the qualities of FCSs to the terahertz region. In a few cases, a link between a continuous wave $(\mathrm{CW})$ terahertz source and a nearinfrared comb has been provided by detection techniques based on photo-conductive antennas ${ }^{13}$ or electro-optic crystals ${ }^{14}$. To detect and phase-lock the beat note, both these techniques involve the $\mathrm{CW}$ terahertz source in a low-efficiency up-conversion process. As a consequence, the CW-source power used for the phase-lock is larger than $1 \mathrm{~mW}$.

Such limitations can be overcome by producing a free-space terahertz FCS and by directly beating it with the CW terahertz source on a square-law detector. This experimental configuration allows independent optimization of the source and reference figures of merit, for an efficient beat-note detection. Once an appropriate detector is used, the amount of power needed for the frequency control of the terahertz source can be dramatically reduced, while making almost the whole power available for the experiment.

The advent of terahertz quantum cascade lasers (QCLs) ${ }^{15}$ is strongly motivating the development of terahertz metrological references. Indeed, as very recent studies have shown, QCLs exhibit a particularly high spectral purity (that is, narrow intrinsic linewidth $)^{16,17}$, which further qualifies them as ideal metrological sources. On the other hand, although terahertz QCLs can presently deliver up to a few hundred milliwatt peak power at very low temperatures $(10 \mathrm{~K})$, a significant reduction of the output power levels is expected when temperatures $>150 \mathrm{~K}$ are approached ${ }^{18}$. From this perspective, the development of a power-efficient metrological referencing technique is therefore very promising.

In this work, we demonstrate a free-space terahertz comb as a mature metrological tool. Indeed, we show that a $2.5 \mathrm{THz}$ QCL can be directly beaten and phase-locked using a commercial hot-electron bolometer (HEB) detector, with only a few tens of nanowatt radiation power required.

\section{Results}

Terahertz FCS generation. The presented terahertz FCS is based on a design commonly used for terahertz time-domain spectroscopy (TDS $)^{19}$. In fact, regardless of the adopted emitter and configuration (antenna, crystal, etc.), any pulsed source of this type is inherently a zero-offset terahertz FCS. Nevertheless, only recently the 'comb' nature of such sources has been recognized and exploited ${ }^{20,21}$, and no direct use of a terahertz comb as frequency 'ruler' for a terahertz laser has been reported so far.

Our terahertz comb aims for a good trade-off between generation efficiency and spectral bandwidth. It is generated by optical rectification, based on the Cherenkov effect ${ }^{22,23}$ of a femtosecond modelocked Ti:sapphire laser in a waveguide fabricated on a MgO-doped $\mathrm{LiNbO}_{3}$ crystal plate ${ }^{24,25}$. The single-mode linear waveguide, optimized for $800 \mathrm{~nm}$ wavelength propagation, is obtained by ion implantation onto the upper surface of the crystal plate. The generation scheme is sketched in Fig. 1a (see Methods for further details). The beam of the pump laser is focused into the waveguide by an aspheric lens. The waveguide lies just below one of the plate surfaces, thus minimizing absorption of the terahertz light within the crystal. We combined, in a non-standard approach, this linear waveguide design with a $45^{\circ}$ high resistivity float zone silicon prism, in contact with the upper crystal surface, for efficiently extracting the train of terahertz pulses $^{26}$. Each pulse consists of a single electric field cycle and carries a large spectral content, from $100 \mathrm{GHz}$ up to $6 \mathrm{THz}$, centred at $1.6 \mathrm{THz}$ (Fig. 1b). As the pulses are identical and equally spaced, the comb-like spectrum of the infinite train has a perfectly zero offset a

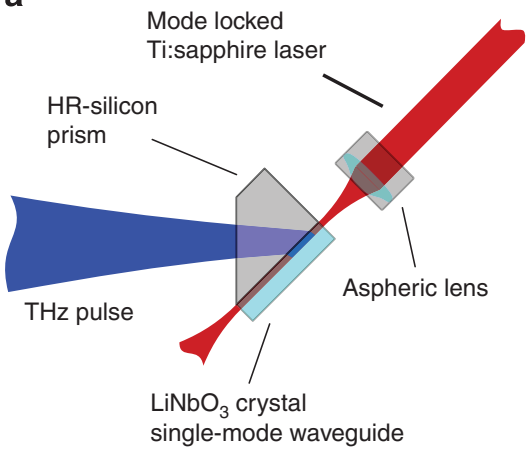

b

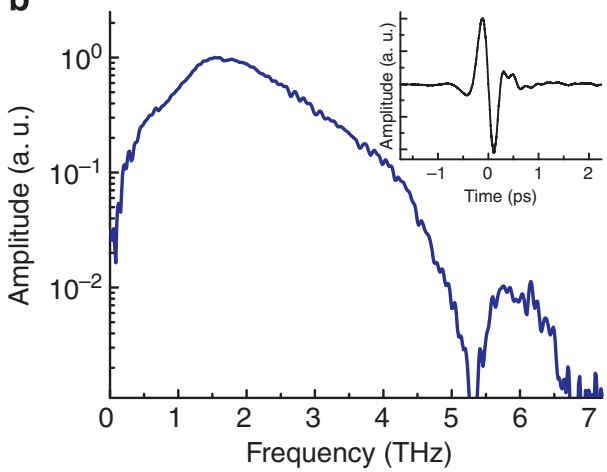

Figure 1 | Generation of the terahertz FCS. (a) Generation scheme of the terahertz pulses based on Cherenkov emission in a lithium niobate waveguide. (b) Terahertz comb spectral extension and single pulse temporal profile (inset) as measured with time-domain spectroscopy technique, using a photoconductive antenna as detector. The fast generation process, taking place in the $\mathrm{LiNbO}_{3}$ crystal, enables extension of the frequency range up to $6 \mathrm{THz}$. The 'comb-like' feature arises from the repetition of identical pulses equally spaced in time. 


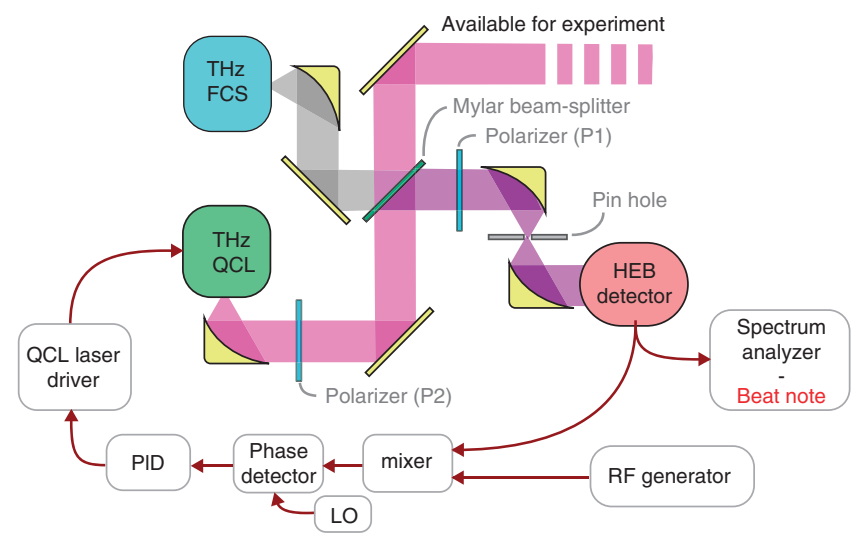

Figure 2 | Beat-note detection and phase-locking setup. The beating between the QCL and the FCS is detected on a HEB. The two beams are superimposed by means of a highly asymmetrical beam splitter, so that more than $99.5 \%$ of the $\mathrm{QCL}$ radiation is available for the experiment beam. The $\mathrm{P} 1$ polarizer ensures polarization matching between the beams, whereas P2 selects the amount of QCL power to be sent to the $\mathrm{HEB}$. Indeed, the QCL terahertz radiation also works as local heater for the superconductive junction, bringing it at the suitable operating temperature. A sketch of the electronic setup is given in the lower part of the panel. The beat note is mixed with a synthesized RF signal, so that it is down-converted to the $21.4 \mathrm{MHz}$ frequency of the phase-lock electronics local oscillator (LO). The output of the phase detector is processed by a standard proportional-integral-derivative (PID) unit and then sent to the $\mathrm{QCL}$ current driver, to close the locking loop.

and a spacing corresponding to the $77.47 \mathrm{MHz}$ repetition rate of the pump laser. In the best conditions, the average power of the generated terahertz radiation is of the order of $1 \mu \mathrm{W}$, as measured by a calibrated pyroelectric detector placed in front of the silicon prism.

Optical setup for beat-note detection and analysis. We use a simple setup (Fig. 2) and a commercial HEB detector to directly observe the beat note between individual comb teeth and a small fraction of the terahertz QCL emission. The QCL, emitting at a frequency around $2.5 \mathrm{THz}$ (see Methods), is housed in a liquid helium cryostat, and driven in continuous-wave mode at a fixed heat sink temperature $T_{\mathrm{O}} \approx 47.5 \mathrm{~K}$. Under these experimental conditions the QCL threshold current is $I_{\text {th }}=375 \mathrm{~mA}$, and the output power corresponding to the operating current $I_{\mathrm{o}}=430 \mathrm{~mA}$ is $P_{\text {out }} \approx 1 \mathrm{~mW}$.

Both the QCL and the pulsed terahertz beams were collected and collimated by $90^{\circ}$ off-axis parabolic mirrors with an equivalent focal length of $25.4 \mathrm{~mm}$. The two beams are then coupled by a mylar film which, at the given wavelength, works as an asymmetric beam splitter, so that most of the terahertz comb power and a small fraction of the QCL beam are overlapped and sent to the HEB. Optical coupling to the HEB is obtained by tuning collimation and diameter of the two beams by means of two $90^{\circ}$ off-axis confocal parabolic mirrors. A pin hole, positioned in their focal plane, provides the required spatial mode cleaning, while a wire-grid polarizer ensures polarization matching.

The optical powers of the QCL and the terahertz FCS impinging on the HEB have been measured by a calibrated pyroelectric detector. In the optimized conditions we have about $60 \mathrm{nW}$ from the QCL beating with about $200 \mathrm{fW}$ of the single comb tooth. Given the $70 \mathrm{fW}$ noise-equivalent power (NEP) of the HEB, we expect a maximum beat note contrast of about $60 \mathrm{~dB}$, in a $1 \mathrm{~Hz}$ bandwidth.

The HEB $250 \mathrm{MHz}$ electrical bandwidth (see Methods) provides a wide frequency window where the beat note can be observed. In fact, as the spacing between the terahertz comb teeth is $77.47 \mathrm{MHz}$, there always are several beat notes falling within the detection
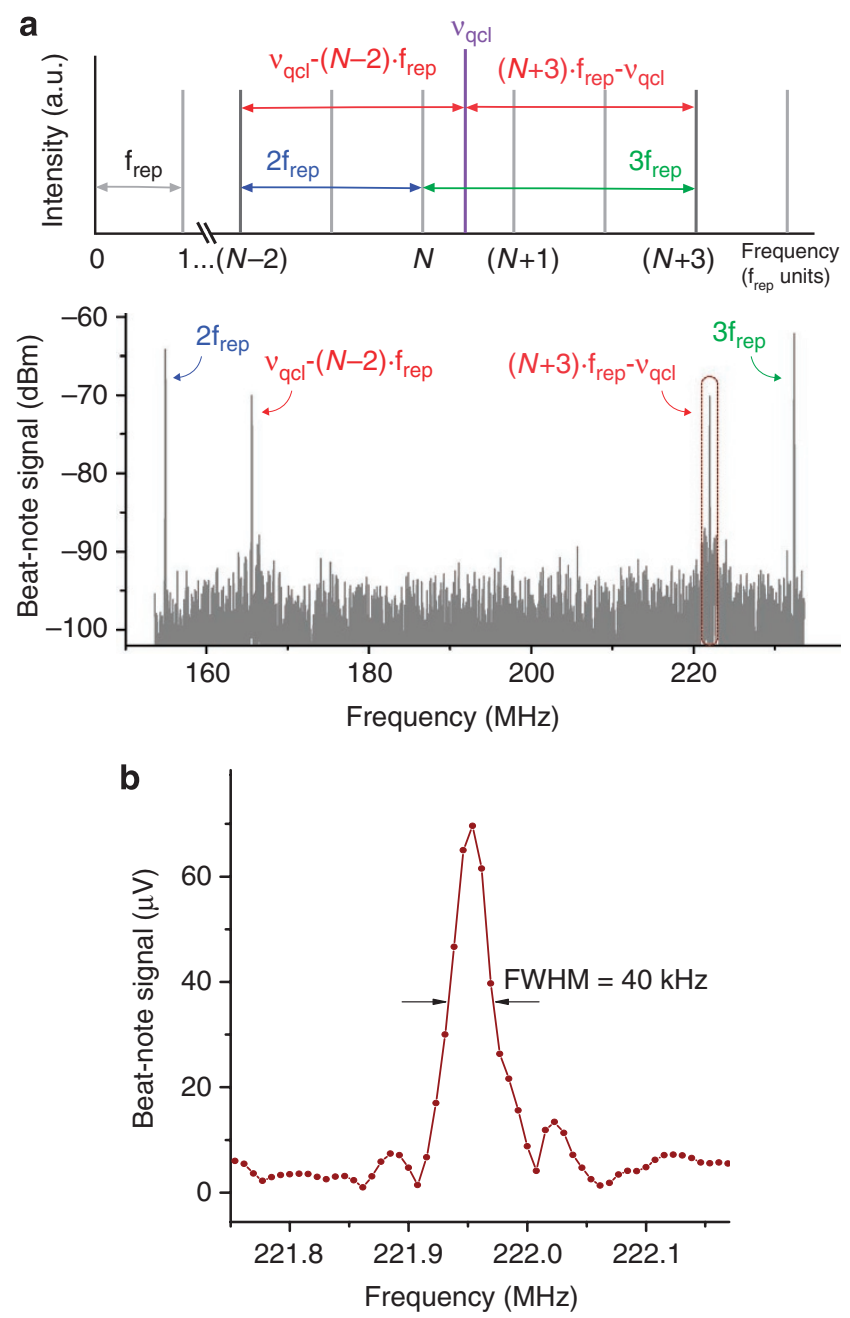

Figure 3 | Beat-note detection. (a) The upper sketch shows, in the frequency domain, the comb-like structure of the terahertz FCS beam, as well as the monochromatic QCL emission $\left(v_{\mathrm{qcl}}\right)$. The red-coloured arrows indicate the beating between the QCL and the third-order neighbours teeth of the comb. The corresponding frequencies fall in the range between $2 f_{\text {rep }}$ and $3 f_{\text {rep }}$ (blue and green arrows, respectively), and are shown in the FFT spectrum below, together with the self-beatings among the comb teeth (as highlighted by the coloured labels). The beat-note frequency is given, apart from $f_{\text {rep }}$ multiples, by its distance from the closest $N^{\text {th }}$ and $(N+1)^{\text {th }}$ teeth of the comb. Therefore, once the order $N$ is known, the QCL absolute frequency can be retrieved. (b) Zoomed view of the beat-note peak around $222 \mathrm{MHz}$ (dashed box in a), acquired with a $20 \mathrm{kHz}$ RBW. The $40 \mathrm{kHz}$ FWHM gives the emission linewidth of the free-running QCL over $1 \mathrm{~ms}$ observation time.

bandwidth. This allows to detect higher-order beat notes, and gives the possibility to select the Fourier interval where a lower background noise maximizes the signal-to-noise ratio.

The signal obtained from the HEB detector is split and sent to a fast Fourier transform (FFT) real-time spectrum analyser (see Methods for further details) for the beat-note measurements, and to the phase-lock electronics.

A typical FFT spectrum of the detected signal is shown in Fig. 3a, together with a schematic sketch of the observed frequencies. The spectrum shows both a pair of peaks spaced by $77.47 \mathrm{MHz}$, arising from the beating of comb teeth among themselves, and a pair of smaller peaks, generated by the beating of the QCL with two distinct comb teeth, one at its left and one at its right. In the case shown, they correspond to the beating with the third-order 

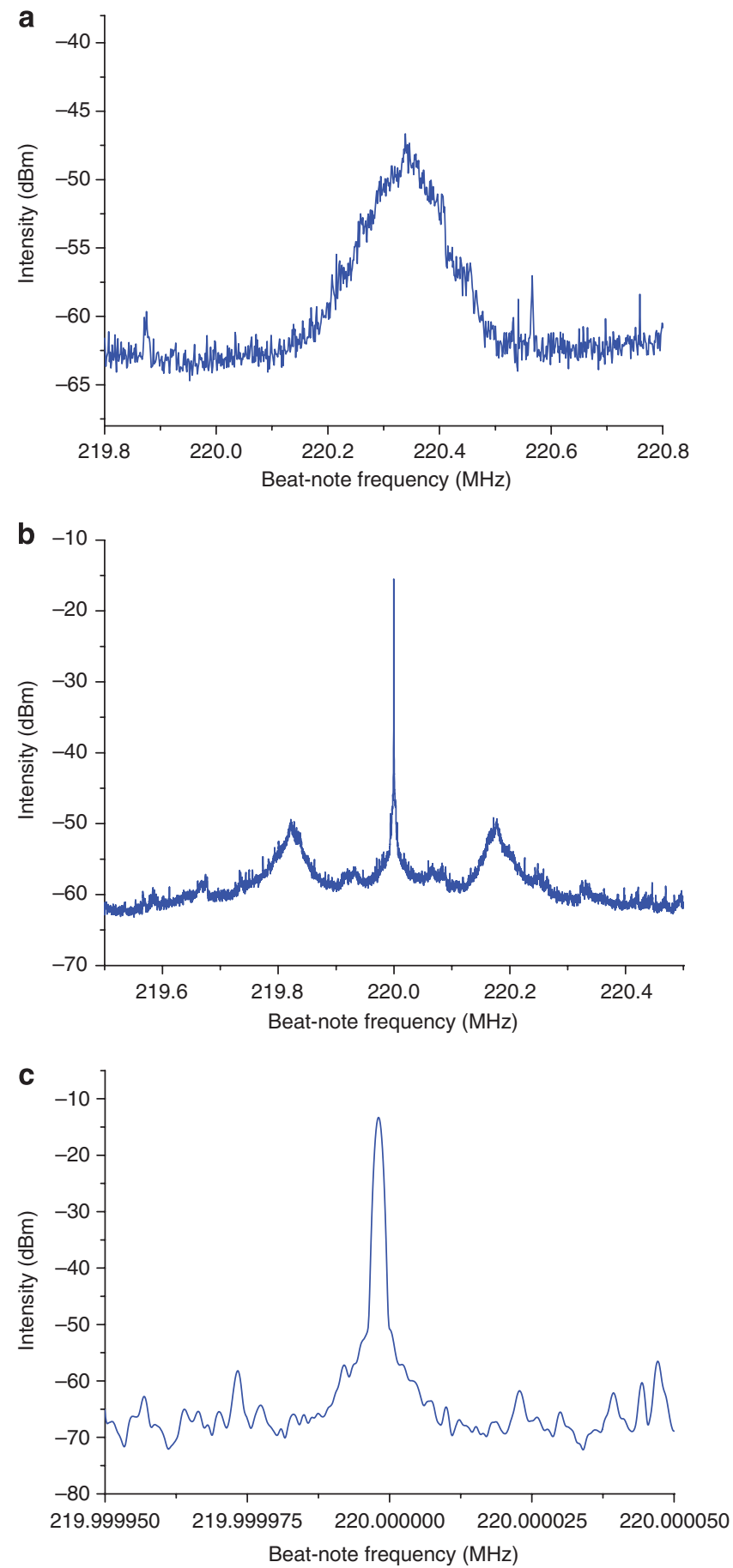

Figure 4 | FFT spectrum analyzer acquisition spectra. The lineshape of the beat-note spectra gives direct evidence of the effects of the phaselock on the QCL emission. (a) Unlocked QCL, with $1 \mathrm{MHz}$ span and $100 \mathrm{~Hz}$ RBW. (b) Phase-locked beat-note signal acquired with a $1 \mathrm{MHz}$ span and $100 \mathrm{~Hz}$ RBW. The two sidebands indicate a phase-lock electronic bandwidth of about $200 \mathrm{kHz}$. (c) Phase-locked beat-note signal acquired with a $100 \mathrm{~Hz}$ span and $1 \mathrm{~Hz}$ RBW.

neighbours teeth. Once the order of the involved comb tooth is known, the measurement of the beat-note frequency can be used to retrieve the QCL absolute frequency, thus enabling to count it and to trace its temporal evolution in real time.

The FFT spectrum analyzer is also used to retrieve the width of the beat note at different timescales. Figure $3 \mathrm{~b}$ shows the beat-note peak over a single FFT spectrum acquisition time ( $1 \mathrm{~ms})$.
The measured full width half maximum (FWHM) of about $40 \mathrm{kHz}$ is in good agreement with previous independent results ${ }^{27,28}$ and with our previous measurements of the frequency-noise power spectral density of the same device ${ }^{17}$. It provides a quantitative estimation of the spectral purity of the laser emission over a $1 \mathrm{~ms}$ timescale, whereas on longer timescales the jitter of the QCL frequency obviously leads to broader signals. Figure 4a shows the $250 \mathrm{kHz}$ FWHM of the free-running beat note, analysed over a $100 \mathrm{~ms}$ timescale.

Phase-lock between terahertz QCL and FCS. To effectively stabilize the phase of the QCL emission to the frequency comb reference, a phase-lock loop (PLL) has been implemented. The simplified scheme of the electronic setup used for closing the PLL is given in Fig. 2. The beat-note signal is mixed with a synthesized fixed frequency and processed by an analog/digital phase detector. The correction signal closes the PLL on the fast ( $1 \mathrm{MHz}$ bandwidth) modulation input of our home-made low-noise QCL current driver. Locked beat-note spectra at different spans and resolution bandwidths (RBWs) are shown in Fig. 4b,c. The electronic bandwidth of the loop is about $200 \mathrm{kHz}$, and the achieved signal-to-noise ratio is $>50 \mathrm{~dB}$ at $1 \mathrm{~Hz}$ RBW, close to the expected limit of $60 \mathrm{~dB}$. By numerical integration of the beat-note spectra, we find that about $75 \%$ of the QCL power is phase-locked to the FCS emission.

The phase-lock leads to a narrowing of most of the CW laser emission down to the terahertz comb tooth linewidth. As shown by Yasui et al. ${ }^{20}$, the linewidth of a terahertz photo-carrier comb (at $100 \mathrm{GHz}$ ) is just few hertz wide (in 1s). Similar results can be achieved by our system, once proper stabilization of the pump laser repetition rate is set.

Beyond the observed performances, ample room is left for additional improvements to further enhance the beat-note signal-tonoise ratio. One relies on proper spectral filtering of the broad-band terahertz FCS that would reduce the portion of power contributing to noise but not to the beating. The second is the optimization of the comb generation process. In particular, a chirped waveguide design can be developed to compensate pump pulse dispersion along its propagation, thus increasing the crystal volume involved in generation. Moreover, the development of new high-bandwidth, low-NEP, terahertz detectors, such as nanowire field-effect transistors ${ }^{29,30}$, could bring, in the next future, to a room-temperature detection of the beat-note signal.

\section{Discussion}

In conclusion, we demonstrate phase locking of a $2.5 \mathrm{THz}$ QCL to a 6-octaves frequency-spanning air-propagating terahertz FCS. The $\mathrm{CW}$ terahertz power required for the phase-lock is four orders of magnitude lower than in other previous approaches. The proposed technique will therefore have a key role in experiments where only a negligible fraction of the terahertz source power is available for phase/frequency control. This paves the way to new experiments and applications in the terahertz range, such as absolute frequency measurements of sub-Doppler molecular spectra, phase locking of high-temperature terahertz QCLs, or manipulation and control of cold molecules.

\section{Methods}

Crystal plate design. The $\mathrm{MgO}$-doped $\mathrm{LiNbO}_{3}$ crystal plate, purchased from the HC-Photonics company, has the dimensions of $8 \times 10 \times 0.5 \mathrm{~mm}^{3}$. It holds two linear waveguides, obtained by ion implantation on the same crystal surface, oriented along the $10 \mathrm{~mm}$-long side. Their design is optimized for single-mode propagation of a $800 \mathrm{~nm}$ radiation, with an effective mode size of about $5 \mu \mathrm{m}$ diameter. According to the Cherenkov configuration, the orientations of the optical field polarization and the crystal optical axis have been chosen both parallel to the crystal plate surface and orthogonal to the waveguide input surface. This choice allows the maximization of the terahertz emission outgoing from the plate surfaces.

Pump laser source. The pump laser is a self-mode-locked Ti:sapphire laser (by Femtolasers, mod. Fusion) emitting pulses with $25 \mathrm{fs}$ temporal width, $77.47 \mathrm{MHz}$ 
repetition rate, around $800 \mathrm{~nm}$ wavelength. As the repetition rate is not stabilized, it is affected by slow drifts of the order of hundreds of millihertz in $1 \mathrm{~s}$. This instability does not affect the measurements discussed in the paper, as the observation time of the beat note is much shorter than $1 \mathrm{~s}$.

HEB detector. The commercial detector used for the beat-note acquisition is a HEB detector by Scontel, model RS 0.3-3T-1, whose sensitive element is a $6 \mathrm{~nm}$-thick MoRe bridge. The active area of the sensitive element is connected to a log-spiral antenna, whose main lobe is coupled with a silicon lens. The HEB chip and the lens are mounted on the bolometer holder of a liquid helium cryostat, while the signal radiation is received through a high density polyethylene window. A low-noise cryogenic amplifier, with a $250 \mathrm{MHz}$ bandwidth, is housed inside the same cryostat. Its output signal is directly processed by our real-time spectrum analyzer (see below). A local heater is used for keeping the HEB temperature just below the superconductive transition of the junction. During all the measurements presented in this paper, however, the local heater is switched off, as the QCL radiation itself works as local heater. To allow a fine regulation of the QCL intensity and, consequently, of the HEB junction temperature, a second polarizer has been added along the QCL beam path (labelled as P2 in Fig. 2).

Real-time spectrum analysis. The spectral analysis of the beat-note signal is carried out by a real-time spectrum analyzer (Tektronix, model RSA5106A). The signal, in the time domain, is digitized at 14 bits and at a $300 \mathrm{Ms} \mathrm{s}^{-1}$ sampling rate. A FFT operation is then applied to consecutive sets of the temporal trace, thus allowing to reconstruct the temporal evolution of the signal spectrum.

QCL fabrication procedure. The investigated device is a QCL operating at $2.5 \mathrm{THz}$ and based on a bound-to-continuum design. The heterostructure has been grown by molecular beam epitaxy employing a GaAs/ $/ \mathrm{Al}_{0.15} \mathrm{Ga}_{0.85} \mathrm{As}$ heterostructure ${ }^{14}$ on a nominally undoped GaAs substrate. The waveguide core is formed by 110 repetitions of the gain medium that creates a $14 \mu \mathrm{m}$ thick active region, while the confinement of the emitted light is ensured by a buried highly doped GaAs layer with negative dielectric constant. Devices were processed into $150 \mu \mathrm{m}$ wide laser ridges by optical lithography, with ohmic contact deposition and with a standard surface-plasmon waveguide ${ }^{14}$. Lateral contacts to the buried highly doped layer were kept $30 \mu \mathrm{m}$ away from the ridge side, to avoid too much coupling with the optical mode. The waveguide was completed by the evaporation of $\mathrm{Cr} / \mathrm{Au}$ on top of the ridge to create the main surface-plasmon interface. The lasers were then soldered to a copper bar, wire bonded and mounted on the cold finger of a liquid helium cryostat.

\section{References}

1. Hübers, H. W. et al. High-resolution gas phase spectroscopy with a distributed feedback terahertz quantum cascade laser. Appl. Phys. Lett. 89, 061115 (2006).

2. Bellini, M. et al. Coherent FIR spectroscopy of molecules of atmospheric interest. Infr. Phys. Tech. 36, 37-44 (1995).

3. Hübers, H. W. Terahertz heterodyne receivers. IEEE J. Sel. Top. Quantum Electron. 14, 378-391 (2008).

4. Tonouchi, M. et al. Cutting edge terahertz technology. Nat. Photon. 1, 97-105 (2007).

5. Carr, L. D., DeMille, D., Krems, R. V. \& Ye, J. Cold and ultracold molecules: science, technology, and applications. New. J. Phys. 11, 055049 (2009).

6. Evenson, K. M., Jennings, D. A. \& Petersen, F. R. Tunable far-infrared spectroscopy. Appl. Phys. Lett. 44, 576-579 (1984).

7. Zink, L. R. et al. The rotational far infrared spectrum of ${ }^{13} \mathrm{CO}$. J. Mol. Spectr. 143, 304-310 (1990).

8. Hindle, F. et al. Widely tunable THz synthesizer. Appl. Phys. B Lasers Opt. 104, 763-768 (2011).

9. Mouret, G. et al. THz photomixing synthesizer based on a fiber frequency comb. Opt. Express 17, 22031-22040 (2009).

10. Diddams, S. A. et al. Direct link between microwave and optical frequencies with a $300 \mathrm{THz}$ femtosecond laser comb. Phys. Rev. Lett. 84, 5102-5105 (2000).

11. Maddaloni, P., Cancio, P. \& De Natale, P. Optical comb generators for laser frequency measurement. Meas. Sci. Technol. 20, 052001 (2009).

12. Malara, P., Maddaloni, P., Gagliardi, G. \& De Natale, P. Absolute frequency measurement of molecular transitions by a direct link to a comb generated around 3- $\mu \mathrm{m}$. Opt. Express 16, 8242-8249 (2008).
13. Ravaro, M. et al. Phase-locking of a $2.5 \mathrm{THz}$ quantum cascade laser to a frequency comb using a GaAs photomixer. Opt. Lett. 36, 3969-3971 (2011).

14. Barbieri, S. et al. Phase-locking of a $2.7-\mathrm{THz}$ quantum cascade laser to a modelocked erbium-doped fibre laser. Nat. Photon. 4, 636-640 (2010).

15. Kohler, R. et al. Terahertz semiconductor-heterostructure laser. Nature 417, 156-159 (2002).

16. Bartalini, S. et al. Observing the intrinsic linewidth of a quantum-cascade laser: beyond the Schawlow-Townes limit. Phys. Rev. Lett. 104, 083904 (2010).

17. Vitiello, M. S. et al. Quantum-limited frequency fluctuations in a terahertz laser. Nat. Photon. 6, 525-528 (2012).

18. Williams, B. S. Terahertz quantum-cascade lasers. Nat. Photon. 1, 517-525 (2007).

19. van Exter, M., Fattinger, C. \& Grischkowsky, D. R. Terahertz time-domain spectroscopy of water vapor. Opt. Lett. 14, 1128-1130 (1989).

20. Yasui, T. et al. Terahertz frequency metrology based on frequency comb. IEEE J. Sel. Top. Quantum Electron. 17, 191-201 (2011).

21. Klatt, G., Gebs, R., Janke, C., Dekorsy, T. \& Bartels, A. Rapid-scanning terahertz precision spectrometer with more than $6 \mathrm{THz}$ spectral coverage. Opt. Express 17, 22847-22854 (2009).

22. Askaryan, G. A. Cherenkov and transition radiation from electromagnetic waves. ZhETF 42, 1360 (1962).

23. Auston, D. N., Cheung, K. P., Valdmanis, J. A. \& Kleinman, D. A. Cherenkov radiation from femtosecond optical pulses in electro-optic media. Phys. Rev. Lett. 53, 1555-1558 (1984).

24. Bodrov, S. B. et al. Highly efficient optical-to-terahertz conversion in a sandwich structure with $\mathrm{LiNbO}_{3}$ core. Opt. Express 17, 1871-1879 (2009).

25. Staus, C., Kuech, T. \& Mc Caughan, L. Continuously phase-matched terahertz difference frequency generation in an embedded-waveguide structure supporting only fundamental modes. Opt. Express 16, 13296-13303 (2008).

26. Theuer, M. et al. Efficient generation of Cherenkov-type terahertz radiation from a lithium niobate crystal with a silicon prism output coupler. Appl. Phys. Lett. 88, 071122 (2006).

27. Barkan, A. et al. Linewidth and tuning characteristics of terahertz quantum cascade lasers. Opt. Lett. 29, 575-577 (2004).

28. Barbieri, S. et al. Heterodyne mixing of two far-infrared quantum cascade lasers by use of a point-contact Schottky diode. Opt. Lett. 29, 1632-1634 (2004).

29. Vitiello, M. S. et al. Room-temperature terahertz detectors based on semiconductor nanowire field-effect transistors. Nano Lett. 12, 96-101 (2012).

30. Vitiello, M. S. et al. Semiconductor nanowires for highly sensitive, roomtemperature detection of terahertz quantum cascade laser emission. Appl. Phys. Lett. 100, 241101 (2012).

\section{Acknowledgements}

This research has been performed at LENS with the support of an EC grant No. RII3-CT2003-506350 and COST Action MP0902-COINAPO. It was partly funded by Ente Cassa di Risparmio di Firenze and by Regione Toscana, through the project CTOTUS, in the framework of POR-CReO FESR 2007-2013 and by the Italian Ministry of Education, University and Research (MIUR) through the program 'FIRB-Futuro in Ricerca 2010' RBFR10LULP 'Fundamental research on terahertz photonic devices'. We acknowledge G. Ferrari for his valuable contribution to the waveguide design and L. Mahler for the QCL fabrication. We also thank A. Montori for the development of the QCL current driver and N. Poli for lending a tunable filter.

\section{Author contributions}

All authors have made significant contributions to the paper. More specific contributions are listed as follows. Experiment planning: S.B., L.C., P.D.N., P.B., R.T. Experimental set-up: A. Ta., P.B., L C., S.B., P.C., M.S.V., A. Tr. Growth of QCL heterostructure: H.E.B. and D.A.R. Data interpretation and writing of the manuscript: L.C., S.B., M.S.V. and P.D.N. with discussion and contributions from all the authors.

\section{Additional information}

Competing financial interests: The authors declare no competing financial interests.

Reprints and permission information is available online at http://npg.nature.com/ reprintsandpermissions/

How to cite this article: Consolino, L. et al. Phase-locking to a free-space terahertz comb for metrological-grade terahertz lasers. Nat. Commun. 3:1040 doi: 10.1038/ncomms2048 (2012). 